SI SOMOS AMIERICANOS

Revista de Estudios

Transfronterizos
Volumen XX, número 1,

enero-junio de 2020.

Recibido: 25 de noviembre 2018.

Aprobado:16 de febrero de 2020.

\title{
Relaciones bilaterales Japón-Chile (1990-1997): contra la incertidumbre, la alianza estratégica*
}

\section{Bilateral Japan-Chile relations (1990-1997): Against uncertainty, the strategic alliance}

\author{
Lenissett Toro Ibacache** \\ Instituto de Estudios Avanzados (IDEA), Universidad de Santiago de Chile, \\ Santiago, Chile.
}

\section{Resumen}

El objetivo de este artículo es entender y explicar los factores que impulsaron que una potencia económica como Japón propusiera una alianza estratégica a Chile en 1994. La hipótesis es que la estabilidad económica chilena de los noventas y la oportunidad japonesa de acceder a las economías del MERCOSUR impulsaron esta alianza. Sin embargo, la crisis económica del Este asiático de 1997, que promovió el reemplazo de Japón por China como mercado para la exportación latinoamericana en Asia, interfirió para que esta idea no se concretara en su diseño original. Para ello se construyó un patrón de jerarquización de visitas entre ambos países que evidenció la brecha entre la agenda de política exterior y los intereses del empresariado chileno.

Palabras clave: Chile, Japón, historia de las relaciones internacionales.

* Este artículo contó con el financiamiento del Fondecyt Regular No 1140244.

** Periodista, Universidad Las Condes. Magíster en Estudios Internacionales, Universidad de Santiago de Chile (Usach). Doctoranda en Estudios Americanos, Usach. Correo electrónico: lenissett.toro@usach.cl

Cómo citar este artículo: Toro, L. (2020). Relaciones bilaterales Japón-Chile (1990-1997): contra la incertidumbre, la alianza estratégica. Si Somos Americanos. Revista de Estudios Transfronterizos, 20(1), 55-77. doi: 10.4067/S0719-09482020000100055 


\begin{abstract}
This paper aims to understand and explain the factors that prompted an economic powerhouse like Japan to propose a strategic alliance with Chile in 1994. The hypothesis is that Chilean financial stability in the 1990s and an opportunity for the Japanese to access the MERCOSUR economies were the factors that drove this alliance. However, the impact of the Asian financial crisis in 1997, which led Japan to be replaced by China as the primary market for Latin American exports in Asia, prevented this idea from materializing as it was originally meant to. In response, a series of high-level official visits took place between the countries, which highlighted the gap between Chile's foreign policy agenda and the interests of the country's business sector.
\end{abstract}

Keywords: Chile, Japan, History of International Relations.

\title{
Introducción
}

Las relaciones internacionales asimétricas han sido estudiadas desde las perspectivas de las externalidades negativas de ellas, expresadas en relaciones de subordinación y hegemonía (teorías del imperialismo y cepalinas). Su teorización concibe las relaciones asimétricas como vínculos basados en esquemas de interdependencia regresiva ${ }^{1}$ que redunda en un perjuicio para el actor más débil (Keohane y Nye, 1977).

Las relaciones de las grandes potencias económicas con los países de América del Sur se abordaron con el mismo tipo de enfoque, países con pocas ventajas competitivas y condicionados por el desarrollo de otros países. No obstante, los procesos de globalización de la década de 1990 abrieron paso a otro tipo de relaciones en las que, a pesar de las diferencias de desarrollo, proporcionaron beneficios a algunos países.

Este trabajo se ocupa de entender y explicar (Hollis y Smith, 1990) el vínculo entre Japón y Chile, dos actores que han desarrollado una relación de "asimetría virtuosa" y que, a pesar de contar con un poder desigual, han logrado establecer un vínculo de "suma variable" mediante el cual ambos se ven beneficiados (Ross, 2007; Ross y Leiva, 2017).

En el marco de este tipo de relación, Japón trató de impulsar una alianza estratégica compleja con Chile (en 1994) que incluía aspectos políticos, económicos y académicos, la cual debía convertirse en un puente entre los países del Asia Pacífico y los del MERCOSUR (Wilhelmy y Lazo, 1997; Gutiérrez, 2005).

1. Se refiere a aquella asimetría que redunda en un perjuicio para el actor más débil, cuya interacción redunda en un desempeño crítico que, pese a estar sujeto a avances y retrocesos, en la suma total siempre culmina en un retroceso (Ross y Leiva, 2017). 
En la base de la propuesta estaba el interés del Gobierno japonés por dinamizar su economía y potenciar el intercambio político y académico, mientras que el chileno buscaba avanzar en su reinserción internacional. Esto movilizó a los gobiernos, empresariados y profesores de ambos países a empujar un vínculo estratégico, cuya desventaja inicial fue la crisis económica japonesa. No obstante, en el mismo período que los gobiernos chilenos trabajaban fuertemente en la reinserción política y económica, Japón ingresó a uno de inestabilidad que dilató las conversaciones y cuya consecuencia inevitable fue el repliegue del plan estratégico bilateral y la idea del puente entre ambos países.

Finalmente, la idea no se materializó y Chile perdió la oportunidad de configurar la mayor alianza que un país en vías de desarrollo podría haber tenido con la segunda economía del mundo, ocasión que a Chile no se le había presentado antes y que le hubiese permitido tomar desafíos que solo podría haber abordado teniendo a Japón como socio estratégico. ${ }^{2}$ En este contexto, el ascenso de China se convirtió en una atracción económica muy poderosa para los países latinoamericanos y Chile no fue la excepción.

Si bien el objetivo de firmar un Tratado de Libre Comercio (TLC) se alcanzó en el 2007, las condiciones económicas de la década anterior no se repitieron. La pregunta que guía este trabajo es ¿cuáles fueron las variables que impulsaron que una potencia económica como Japón propusiera una alianza estratégica a un país como Chile y cuáles interfirieron para que la idea del puente no se concretara durante el período de estudio?

En busca de respuestas, este trabajo indaga en las claves para entender la propuesta japonesa en un contexto histórico específico de continuidad y cambio. Para ello se consideró la revisión de un marco temporal que se inicia con el fin de la Guerra Fría (1990) y termina con la crisis financiera asiática (1997). Luego se estudió los efectos de estos acontecimientos en la relación entre ambos países, momento en el que Chile iniciaba un proceso democrático y de apertura política, mientras que Japón se encontraba en un período abrupto de cambios políticos y enfrentaba una crisis económica que golpeó fuertemente su posicionamiento internacional.

La hipótesis sostenida aquí es que la estabilidad económica chilena, y las posibilidades japonesas de acceder a las economías del MERCOSUR, impulsaron al Gobierno nipón a proponer una alianza estratégica a Chile. No obstante, la crisis económica que afectó a Japón a inicios de la década de 1990 desaceleró este proceso de negociaciones, lo que, unido al ascenso de China, interfirieron para que la idea del puente no se concretara durante el período de estudio.

Esta investigación se articula en el marco de tres enfoques: historia de las relaciones internacionales, relaciones internacionales e historia del tiempo presente, considerando que

2. Impactos no solo referidos al desarrollo económico, sino también al desarrollo de investigaciones conjuntas, en educación, en transferencia tecnológica e infraestructura, son solo algunos de los aspectos en el que un país como Chile podría haberse beneficiado. 
las fronteras entre historia diplomática, historia internacional, historia mundial/global e historia transnacional son relativamente tenues (Williams, 2012). Se busca problematizar, desde un punto de vista histórico, las claves de la relación entre estos dos países en un lapso de tiempo seleccionado, bajo una perspectiva integradora (Braudel, 2005; Hollis y Smith, 1990; Huguet, 2001).

La metodología que se utiliza es de tipo hipotético-deductivo, a partir de la cual se formula una hipótesis, se explica un fenómeno, para luego someter a prueba la hipótesis. En este caso se comprobará la hipótesis utilizando la metodología de “jerarquización de visitas”, propuesta por el profesor César Ross (Ross, 2018; Ross y Leiva, 2017). Esta permite proponer nuevas conjeturas a partir de la creación de un patrón de visitas entre países. ${ }^{3}$

Para el desarrollo de este trabajo se han utilizados las siguientes fuentes: leyes, tratados, acuerdos, información de las distintas instituciones de los Gobiernos japonés y chileno divulgadas a través de sus sitios web, así como entrevistas a académicos japoneses que estuvieron en el proceso y publicaciones de autores que hacen referencia a las relaciones de Japón con los países de América y en especial con Chile.

En cuanto a su organización, el artículo se inicia describiendo el contexto histórico en el que Japón impulsó las relaciones bilaterales, luego desarrolla la idea e implicancias de una vinculación estratégica, para a continuación aplicar la metodología de jerarquización de visitas que permitirá concluir por qué esta idea se frustró durante el período de estudio.

\section{El impulso inicial: los cambios en la política exterior japonesa}

El fin de la Guerra Fría constituyó un momento crítico para el debate historiográfico y político: ¿cuál sería la forma que tomaría nuestro mundo después de concluida esta fase? Para los países fue un período de incertidumbre que debieron resolver en la medida en que los acontecimientos fueron sucediendo.

Para Japón significó un nuevo desafío para su política exterior de seguridad, ya que su condición de potencia económica provocó que la comunidad internacional, y en especial su aliado, Estados Unidos, le exigiera un rol más decidido en el sistema que se estaba configurando (Toro, 2018). En el ámbito económico, el país nipón estaba viviendo las consecuencias de la burbuja inmobiliaria (1986-1991) que hizo colapsar la economía a partir de los noventa con una desaceleración de las inversiones (1990), continuó con el

3. Una vez anunciada la idea de negociación de las distintas partes, el estudio de las "Memorias" del Ministerio de Relaciones Exteriores de Chile permite revisar la versión oficial del vínculo de nuestro país con otros. Si bien esta fuente no es la única, su estudio permite reconstruir de manera muy pormenorizada el curso del vínculo estudiado. 
colapso de los bancos (1991), una recesión (1992) y la apreciación del yen, lo que acompañado de una inestabilidad política retardó la aplicación de medidas de reactivación. Esto llevó al Gobierno japonés a concluir que, a pesar de su fortaleza económica, era vulnerable a la turbulencia económica y a los cambios políticos, por lo que debía explorar nuevas opciones para mantener su estabilidad. ${ }^{4}$

En este contexto, Japón buscó socios fuera de la región para contrarrestar las consecuencias de la crisis y desarrolló alianzas estratégicas que le permitieran acceder a materias primas imprescindibles. Como resultado de estas transformaciones, propuso acuerdos a dos países latinoamericanos: primero a México, una plataforma comercial hacia Estados Unidos (Japanese Access to Mexico: The Structure of the Japan-México FTA, 2016), y luego Chile, ${ }^{5}$ que era visto como una puerta de entrada a las economías del MERCOSUR (ChileJapón, 1990-2007: Avances y repliegues en una alianza estratégica fallida, 2018).

Pero ¿cuáles fueron los factores que influyeron para que Japón propusiera una alianza estratégica a un país como Chile, cuya diferencia de desarrollo era tan grande? Sobre la base de la información conocida por esta investigación, se identificaron tres factores: a) los cambios en el sistema internacional y los efectos en las alianzas político-económicas; b) la construcción de lazos bilaterales durante el período de la Guerra Fría; c) el empuje hacia la internacionalización y el regionalismo abierto.

\section{Los cambios en el sistema internacional: entre la competencia y la alianza estratégica}

La cooperación redujo la incertidumbre, especialmente en aquellas relaciones de naturaleza asimétrica. Para los Estados, establecer acuerdos fue crucial, siendo uno de los objetivos más importantes en la política exterior, tanto para las grandes potencias como para los pequeños países que vieron en las relaciones de asimetría virtuosa una oportunidad para el desarrollo de sus mercados.

Las "alianzas estratégicas" constituyeron una forma de vinculación mayor con objetivos específicos: primero, conseguir la expansión territorial o de seguridad, como se evidenció desde fines del siglo XIX; segundo, para el desarrollo económico, ${ }^{6}$ especialmente en la segunda mitad del siglo XX y; tercero, para el intercambio de investigación para el

4 Japón no es excepcional como algunos autores afirman. Enfrentó las mismas situaciones que el resto de los países, lo único que lo hizo diferente fue la limitación impuesta por el artículo 9 de la Constitución (que le prohíbe la utilización de armas), por lo que necesita planificar mejor sus respuestas a eventos mundiales para que pueda reaccionar de manera oportuna y apropiada (Cooney, 2015).

5 Esta propuesta está documentada en el Acta del Comité Empresarial Chile-Japón de 1994.

6 Especialmente referido al desarrollo de convenios de primera, segunda y tercera generación. 
desarrollo $(\mathrm{I}+\mathrm{D}){ }^{7}$ aunque sin dejar atrás el modelo más básico de compra y venta de commodities (Dickson y Weaver, 1997; Grant y Baden-Fuller, 2004).

A nivel macro, algunas regiones se volcaron en procesos de integración (cultural y económica) como Europa $^{8}$ y América Latina ${ }^{9}$. En la región asiática, esto implicó un proceso más complejo, ya que los abusos cometidos por el ejército imperial japonés durante la primera mitad del siglo XX hicieron más compleja una integración cultural; no obstante, lograron alianzas económicas concretas exitosas.

El Gobierno japonés utilizó la Ayuda Oficial para el Desarrollo (AOD) ${ }^{10}$ como una fórmula de compensación por los daños causados, pero también para la promoción de la paz para la construcción de un entorno regional libre de amenazas y para crear una cadena de valor con los Estados de la región, donde Japón era el líder económico.

Este proceso de cooperación había comenzado en la década de 1960, cuando los flujos de comercio e inversión entre los países de la Cuenca del Pacífico empezaron a influir en los patrones económicos regionales a medida que crecían las economías de mercado del Pacífico (Marris y Overlana, 1997). Frente a esta realidad, la comunidad empresarial japonesa impulsó el vínculo con socios comerciales clave de la región para formar comités de cooperación económica bilaterales. ${ }^{11}$

En 1967 se creó la Pacific Basin Economic Council (PBEC), ${ }^{12}$ que entre 1978 y 1983 celebró reuniones generales en Filipinas (Manila), Hong Kong y Chile (Santiago), donde se reconoció la importancia de los países recientemente industrializados del ASEAN y América Latina, por lo que se decidió impulsar acuerdos económicos y vínculos estratégicos hacia esas regiones (Marris y Overlana, 1997).

7 Especialmente referido al desarrollo de convenios de cuarta generación. Se trata de vínculos más complejos que comenzaron a evidenciarse a partir de la crisis económica de 1973 y a partir del aumento de la competitividad de Japón y de los países de Asia sudoriental. Este cambio en la forma de vinculación (I+D) significó un salto cuántico respecto a las políticas establecidas después de las dos grandes guerras, que apostaron por la producción a gran escala de las empresas, las que a su vez tenían un comportamiento burocrático y relaciones laborales mínimas, que consideraban la transferencia de tecnología, ya que su objetivo principal era la competencia económica y crear empleos (Casson, 2010).

8 Que devino en la creación de la Unión Europea en 1993.

9 Que devino en la creación del Mercado Común del Sur (MERCOSUR) en 1991.

10 La ayuda económica japonesa se inició en 1954, con un convenio de cooperación con Birmania, Filipinas e Indonesia. Bajo la actual AOD, se inició en la década de 1960, cuando Japón inició su crecimiento económico.

11 En 1961 se creó el Comité Mixto Empresario Argentino-Japonés de 1961. En 1962, se concretó el Comité de Cooperación Empresarial Japón Australia (JABCC, por su sigla en inglés) y su contraparte, el Comité de Cooperación Empresarial Australia Japón (AJBCC). Ambos tenían como objetivo proporcionar un foro en el que los comités empresariales de los dos países pudieran debatir conjuntamente objetivos comerciales y económicos comunes y generar nuevas oportunidades comerciales y de inversión, modelo que luego se replicaría en América Latina.

12 En 1965, los economistas Kiyoshi Kojima y Hiroshi Kurimoto propusieron el Pacific Free Trade Area (PAFTA), acuerdo que involucró a las cinco economías más desarrolladas en el Pacífico: Australia, Canadá, Estados Unidos, Japón y Nueva Zelandia, y que fue el precursor de la Pacific Basin Economic Council (PBEC). 


\section{La construcción de lazos bilaterales durante el período de la Guerra Fría}

El segundo factor que impulsó la idea del puente fue la larga historia de relaciones entre Japón y Chile, iniciada en 1897 con la firma del Tratado de Amistad, Comercio y Navegación, por lo que las bases de esta relación se formaron mucho antes de que las relaciones de Japón con Occidente quedaran bajo la supervisión hegemónica de Estados Unidos, lo que Stallings y Székely (1994) denominaron "trilateralidad". No obstante, investigaciones recientes demuestran que Japón y Chile desarrollaron un camino lateral, distinto al descrito por Stallings y Székely (1994). En ellas se describe cómo la coexistencia de una rutina diplomática y una activa política bilateral permitieron reducir la incertidumbre económica chilena, especialmente a partir de los setenta (Ross, 2013, 2014).

Este camino lateral implicó que los japoneses contradijeran a Washington a cambio de garantizar las materias primas claves en su proyecto industrial, mientras que para Chile significó desarrollar un vínculo pragmático y distanciado de los valores centrales del proyecto histórico de la izquierda chilena de entonces, a cambio de recursos económicos que permitieran financiar las transformaciones revolucionarias que planteaba el Programa de Gobierno de Allende. ${ }^{13}$ Tras el golpe de Estado en septiembre de 1973, ambos países continuaron con su pragmatismo político, dando continuidad a la rutina diplomática y reduciendo al máximo el impacto que implicaba este nuevo cambio institucional. ${ }^{14}$

En el caso de la migración, de acuerdo con el estudio realizado por Masterson y FunadaClassen (2004), el número de japoneses que a fines del siglo XIX llegó a América Latina fue: 11.099 a México, 9.106 a Perú, 4.573 a Brasil, 22 a Argentina y 135 a Chile, cifra que varió en 1941, año en que se registraron: 14.566 en México, 33.067 en Perú, 187.687 en Brasil, 5.398 en Argentina, mientras que en Chile solo se contabilizaron 498. Esta baja inmigración significó una desventaja respecto de otros países de América Latina, donde los descendientes japoneses han sido actores activos en la profundización de las relaciones bilaterales (Laborde, 2006)

\section{El empuje hacia la internacionalización y el regionalismo abierto}

Los líderes japoneses fueron factores de cambio en la política exterior, al tomar decisiones que aceleraron algunos procesos como la discusión sobre el regionalismo abierto y la búsqueda de nuevos socios estratégicos. ${ }^{15}$

13 Durante la década de 1970, Japón tuvo una política de acercamiento a nuestro país. Grandes empresas niponas buscaron asociarse en proyectos atractivos, siendo la minería en torno al hierro y las pesqueras, receptoras de este interés.

14 Durante el período 1974-1987, la inversión japonesa en Chile registró un promedio anual inferior a los cinco millones de dólares, haciéndose sentir su impacto en 1988, cuando subió a 123 millones de dólares, como resultado de la inversión en la empresa minera La Escondida (Valenzuela y Varas, 1993).

15 Un ejemplo de esto fue la decisión del primer ministro japonés, Masayoshi Ōhira (1978-1980), de terminar la década de 1970 impulsando el desarrollo del Pacific Basin Cooperation Study Group (1979), cuya misión era estudiar la posibilidad de una zona de libre comercio que en los ochentas permitió la creación 
Al inicio de la década de 1990, Japón propuso acuerdos a dos países latinoamericanos: México y Chile. En ambos casos, el modelo de negociación siguió el mismo patrón: la conformación de mesas de trabajo tripartito con el Gobierno, empresarios y académicos, diálogos a nivel de trabajo, seguidos de negociaciones con los ministerios. Sin embargo, para concretar la idea de una alianza estratégica con Japón a través de la firma de un tratado de libre comercio (TLC), los socios interesados debían sortear distintas instancias: los políticos nipones eran quienes votaban a favor o en contra de las iniciativas comerciales, los ministerios conducían la política comercial y los burócratas eran los encargados de fomentar relaciones con los grupos de interés (Nagata, 2017; Yoshimatsu, 2006; Kamikubo, 2008). ${ }^{16}$

En el caso chileno, el interés japonés por avanzar en una integración comercial mayor coincidió con la reinserción democrática internacional del país $(1990)^{17}$ y con ello se cruzaron los intereses de ambas naciones: Chile quería ser parte de una estrategia comercial mayor y Japón buscaba acceder a los productos del MERCOSUR y desarrollar una cadena de valor que hizo considerar la idea de ser los extremos de un puente que cruzara el Pacífico.

No obstante, Japón había ingresado en una crisis económica ${ }^{18}$ y política ${ }^{19}$ que ralentizaría su proceso de toma de decisiones, lo que coincidió con la emergencia de una nueva potencia económica en la región, China, que capturó la atención de los países latinoamericanos, dado el tamaño de su mercado, y Chile no fue la excepción (Jarufe, 2018; Rodríguez y Leiva, 2013).

Luego de una larga historia de gestos diplomáticos, se generó una tensión entre los principios (amistad) y los intereses (económicos) de ambos países, situación que fue interfiriendo en la idea de la alianza estratégica, la que no se logró concretar en los términos propuestos por Japón y cuyo proceso se revisará en los siguientes puntos (2 y 3$)$.

del Pacific Economic Cooperation Council (PECC) (Nagatomi, 1988), plataforma que sirvió para crear la Asia-Pacific Economic Cooperation (APEC) en 1989.

16 En el caso de las empresas japonesas en México, el mayor lobby estuvo constituido por las empresas multinacionales del sector manufacturero, que estaban preocupadas por la discriminación del TLCAN sobre la IED (japonesa), lo que hizo cambiar la percepción y la estrategia política del Ministerio de Comercio Internacional e Industria (MITI, por su sigla en inglés) y del Ministerio de Economía (METI, por su sigla en inglés), los que empujaron un cambio en la política comercial que implicaba acuerdos más profundos y que apuntaban a una mayor integración económica, una que se pareciera más a una alianza estratégica (Tanaka, 2002).

17 Tras el fin de la dictadura militar y la llegada del gobierno democrático de Patricio Aylwin.

18 La crisis de burbuja y la crisis financiera asiática de 1997.

19 Tras la caída, en 1993, del partido que se mantenía en el poder desde 1995, el Partido Liberal Democrático. Esto significó un cambio en la correlación de fuerzas, pero también una reforma de la ley electoral. 


\section{Japón y Chile: los extremos de un puente}

Chile fue pionero en la liberalización unilateral del comercio a partir de la década de 1970, mientras que en los noventa la tendencia fue la firma de Acuerdos de Complementación Económica (ACE) y de TLC. Estos procesos de asociación económica fueron impulsados por los nuevos gobiernos democráticos liderados por los presidentes, Patricio Aylwin (1990-1994) y Eduardo Frei (1994-2000), quienes iniciaron una estrategia de reinserción global del país ${ }^{20}$ que derivó en dividendos económicos y en la imagen de uno de los países más estables en el sur de América.

Chile siguió el liderazgo de México en los acuerdos con Estados Unidos, Europa y Japón (Stallings, 2009). No obstante, se debe considerar que México tenía una gran ventaja desde el punto de vista geográfico, dada su cercanía con Estados Unidos. Pese a esta aparente desventaja geográfica, Chile desarrolló su propia estrategia para seducir a las distintas economías (Belém, Damascena y Mendonça, 2015), lo que le permitió firmar una serie de acuerdos con México (1991), ${ }^{21}$ Bolivia (1993), Venezuela (1993), Ecuador (1994), un ACE con los países del MERCOSUR (1996) y un TLC con Canadá (1996).

Las reuniones y gestos bilaterales entre Japón y Chile llamaron la atención de la prensa estadounidense. El New York Times publicó una nota que informaba sobre los fuertes lazos entre ambas economías y se preguntaba cómo los chilenos habían sido capaces de penetrar en el mercado japonés, pues este generalmente se había mostrado cerrado para el norteamericano.

De hecho, a algunos en Washington les preocupa que, al establecer vínculos más estrechos con Chile, Japón esté teniendo una importante posición en América Latina, ampliando su influencia económica y obteniendo potencialmente la mayor proporción de minerales, papel y recursos pesqueros en el próximo siglo. (Nash, 15 de abril de 1993, p. 1)

En esta misma nota se destaca la opinión del entonces presidente de Mitsubishi, Koji Mizuna, quien afirmaba:

Si se mira a Chile, está rodeado de agua y montañas y los chilenos también tienden a ser una sociedad cerrada. Los chilenos son trabajadores honestos y muy tímidos como el japonés. Los chilenos son sentimentales y leales como en Japón. Estamos mucho más cómodos haciendo negocios con Chile que con otros países. (Nash, 15 de abril de 1993, p. 1)

Como se aprecia en la cita, el periódico norteamericano daba cuenta de las nuevas relaciones comerciales de Japón con Chile: la inversión y el flujo comercial habían crecido no solo en el aspecto económico, sino también en factores culturales, de conocimiento

20 Esto, luego de 17 años de dictadura militar del general Augusto Pinochet (1973-1989).

21 Acuerdo que en 1998 derivó en la firma de un TLC Chile-México. 
mutuo y por el establecimiento de reglas que dieron confianza acerca del trato de ambas partes $^{22}$ (Valenzuela y Varas, 1993).

En el marco de estas relaciones de confianza, la idea de Chile como parte del extremo de un puente fue impulsada por el Capítulo Chileno del Comité Empresarial Chile-Japón ${ }^{23}$ y asumida por la contraparte japonesa en 1994 (Comité Empresarial Chile-Japón, 1994). Esta idea sería nuevamente mencionada por el primer ministro japonés, Ryūtarō Hashimoto, en su mensaje de saludo con motivo del centenario de las relaciones bilaterales (1997): "Chile, con la mirada puesta en el siglo XXI, se prepara para constituirse en un puente entre Asia y América del Sur" (Ross, 2001). ${ }^{24}$

Este interés japonés respondía a los resultados que venía obteniendo Chile desde los setenta. Si bien el crecimiento de la producción manufacturera en varios países de América fue relativamente alto en esa década, ${ }^{25}$ se redujo drásticamente durante la primera mitad de la década de 1980 y se recuperó algo en la mayoría de los países seleccionados en la segunda mitad de esa década. No obstante, las políticas macroeconómicas desestabilizadoras, así como los regímenes industriales proteccionistas, obstaculizaron el desarrollo de las industrias manufactureras eficientes y las exportaciones durante un largo período de tiempo en varios países de América del Sur, siendo Chile una excepción (Faust, 2004).

A lo largo de la década de 1990 se firmó una serie de acuerdos marco y de cooperación en áreas de política tales como minería, pesca, gestión de recursos naturales, infraestructura y cooperación para el desarrollo con Japón. Las exportaciones chilenas de 1991 se distribuían principalmente en la Comunidad Económica Europea (32\%), Estados Unidos y Japón (18\%) y en América Latina (14\%). En el caso japonés, la mayor parte de la composición de las exportaciones eran recursos minerales. Esta exclusividad tiene varias explicaciones, entre ellas está el escalonamiento arancelario, las barreras fitosanitarias y, probablemente, el desconocimiento del mercado. En 1994, Chile se incorporó al Foro de Cooperación Económica Asia-Pacífico, más conocido como APEC (por su sigla en inglés de Asia Pacific Economic Cooperation), uniéndose a las 21 economías que representaban el 52\% del PIB y $44 \%$ del comercio global (Burges, 2014). En 1995, Japón se convirtió en el donante de ayuda al desarrollo más importante de Chile y, en 1997, en su socio comercial más importante (Minería Chilena, 2014).

22 Otro factor importante en los noventa fue la estabilidad que mostró Chile en el proceso de transición política.

23 Tales gestiones permitieron el exitoso desarrollo de una alianza público-privada que empujó el desarrollo de las relaciones bilaterales, jugando un rol fundamental en la concreción de las iniciativas entre ambos países. Akio Hosono lo llamó "comité de sabios" (César Ross, Fondecyt No 1140244, 6 de junio de 2017).

24 Ross (2001) afirma que las relaciones entre el Comité y el Gobierno chileno eran las de una verdadera asociación. Su influencia sirvió para aumentar la eficiencia de los viajes oficiales a Japón, así como para modificar la política del Estado de Chile frente a los empresarios japoneses que operaban en el país.

25 Colombia, Costa Rica, Ecuador, El Salvador, Guatemala, Uruguay, Venezuela. 
En este marco dinámico de intercambio, la colaboración entre los gobiernos japonés y chileno y los comités empresariales permitieron reducir los costos de transacción de los vínculos formales. Se establecieron normas que facilitaron el intercambio político, económico y contractual. No obstante, para Mikio Kuwayama -exjefe de la Unidad de Comercio Internacional de la División de Comercio Internacional e Integración de la Comisión Económica para América Latina y el Caribe (CEPAL), que participó en las negociaciones comerciales del TLC con Chile- los países latinoamericanos, incluido Chile, no apreciaron el valor del sector privado japonés en la región:

yo no hablo solo de la asistencia oficial del Gobierno, sino también de las inversiones, como usted habla también de las relaciones de [los] comités, sector público-privado, etc. No hay un reconocimiento suficiente por parte de los países latinoamericanos (César Ross, entrevista, 6 de junio de 2017). ${ }^{26}$

El interés comercial japonés durante este período podría haber sido mayor si la inversión se hubiese centrado en las cadenas de valor, tal como lo hizo en Asia. Al respecto, Kuwayama afirma que esta forma de comercio es la que motivaba (y motiva) el mayor interés por parte de su país. No obstante, según Kuwayama (6 de junio de 2017), para el caso de Chile las relaciones bilaterales se centraron en la venta de productos, especialmente de cobre, que alcanzó la mitad del comercio total.

A continuación, se muestran los resultados de la revisión de las "Memorias" del Ministerio de Relaciones Exteriores de ambos países, en las que se registró una serie de actividades y reuniones que buscaron impulsar el conocimiento mutuo que permitiera la firma de una asociación entre ambas partes. ${ }^{27}$ Es precisamente la revisión de estos documentos los que entregan información adicional respecto a las visitas que marcaron los hitos más importantes en la relación bilateral en el período estudiado.

\section{Jerarquía de las visitas: el valor de los hechos}

Las ciencias sociales y políticas buscan elaborar generalizaciones de validez universal. En el caso de los estudios internacionales, sus generalizaciones están destinadas a comprender y explicar los fenómenos internacionales (Hollis y Smith, 1990); es decir, aquellos que trascienden la realidad nacional y que involucra a dos o más Estados.

26 Entrevista realizada en Tokio en el marco del Fondecyt №1140244 por César Ross. Kuwayama es profesor del Departamento de Política Global, Facultad de Leyes, Universidad de Hosei, Tokio, Japón.

27 Por otra parte, la tarea del Comité Empresarial Chile-Japón también fue la de superar limitaciones informales, referida a los códigos de conducta y convenciones culturales que proporcionaron un marco conceptual basado, principalmente, en el lenguaje para la decodificación conductual del otro, de sus valores, así como de otros factores que influyeron en la relación. 
El profesor Ross propone un ejercicio analítico ${ }^{28}$ para comprender y explicar las relaciones bilaterales a través de patrones conductuales, que se pueden extraer del análisis de las memorias escritas ${ }^{29}$ y el registro de las visitas. ${ }^{30}$

Para el caso de esta investigación, se han considerado las visitas ya que, previo a un acuerdo comercial, estas tienen un alto valor en las relaciones internacionales, en tanto representan la mayor demostración de interés por la contraparte y por los asuntos que involucran. En este sentido, el rango de los visitantes, el tiempo de la visita, así como los resultados concretos que se buscan con la visita, son directamente proporcionales a la valoración que se hace de la contraparte.

Para la realización de un patrón de las visitas, es necesario hacer una jerarquía de estas a través de la cual se asigna un puntaje a las visitas según su importancia (presidentes, senadores, profesionales técnicos, empresarios, académicos, etc.), que va del 1 al $100 .^{31}$ Esta metodología ha sido utilizada por Ross en el artículo "La política de Chile hacia Bolivia, 1990-2009. Ni coordinación política, ni cooperación económica” (2017), en el que visibiliza ciertas conductas estructurales (incluso aquellas que parecían menos visible) que le permitieron formular nuevas conjeturas e hipótesis respecto de las relaciones entre Chile y Bolivia.

Es precisamente esta metodología la que se utilizará para analizar las relaciones bilaterales entre Japón y Chile para el período 1990-1997, en la búsqueda de un patrón que permita

28 Metodología jerarquización de visitas, inscrita recientemente en el registro de propiedad intelectual $\mathrm{N}^{\mathbf{0}}$ 288.558 (19/03/2018) en el Servicio Nacional del Patrimonio Cultural del Ministerio de las Culturas, las Artes y Patrimonio de Chile.

29 Se trata de contactos registrados en comunicaciones escritas, en forma de cartas y notas reversales (un tipo de notas diplomáticas), y las visitas en el marco de encuentros multilaterales y, preferentemente, en reuniones entre representantes de dos países para tratar temas de interés. La comunicación escrita tiene la fortaleza de constituir compromisos, en tanto las contrapartes siempre pueden basarse en las comunicaciones anteriores para reclamar compromisos y cumplimientos futuros.

30 Si bien las visitas están basadas en una comunicación oral y, en consecuencia, no necesariamente deriva en un texto que pueda adquirir el rango que se indica en el párrafo anterior, sí permite un acercamiento personal que usualmente facilita el proceso de negociación y de toma de decisiones. Esto también implica que los malentendidos pueden derivar en una tensión o en un impasse que implique un retroceso en la materia que se estaba tratando.

31 Los jefes de Estado (100, que implicará un 33\% de la ponderación total); secretarios de Estado (ministros) y presidentes de organizaciones estatales y no estatales de nivel nacional (80, 27\%); secretarios de Estado adjuntos (viceministros y/o subsecretarios), autoridades de unidades territoriales mayores (gobernadores, intendentes, etc.), senadores nacionales, embajadores, presidentes de organizaciones estatales y no estatales a nivel de unidades territoriales mayores $(60,20 \%)$; autoridades de unidades territoriales intermedias (gobernadores, autoridades provinciales, etc.), ministros consejeros, cónsules, diputados (unidades territoriales intermedias), presidentes de organizaciones estatales y no estatales a nivel de unidades territoriales intermedias (40, 13\%); autoridades de unidades territoriales menores (alcaldes, intendentes, etc.), presidentes de organizaciones estatales y no estatales a nivel de unidades territoriales menores, rectores de universidades $(20,7 \%)$; y por cada integrante de comitiva, diferente a las autoridades, el puntaje será $3(1 \%)$. 
entender y explicar el fracaso de la alianza estratégica (la idea del puente) durante la década de 1990.

El Gráfico $\mathrm{N}^{\circ} 1$ muestra la dinámica de las visitas de representantes chilenos a Japón, así como el impacto de distintas visitas japonesas a Chile, lo que permitió establecer la existencia de tres períodos con características específicas: avance de las relaciones bilaterales (1990-1993), intensificación de la cooperación (1994-1995) y repliegue de las relaciones bilaterales (1996-1997).

\section{Gráfico $N^{\circ}$ 1: Jerarquía de visitas (1990-1997). Visitas según rango por año}

(En el eje Y, el resultado con la suma de las visitas, y en el eje X, los años en estudio)

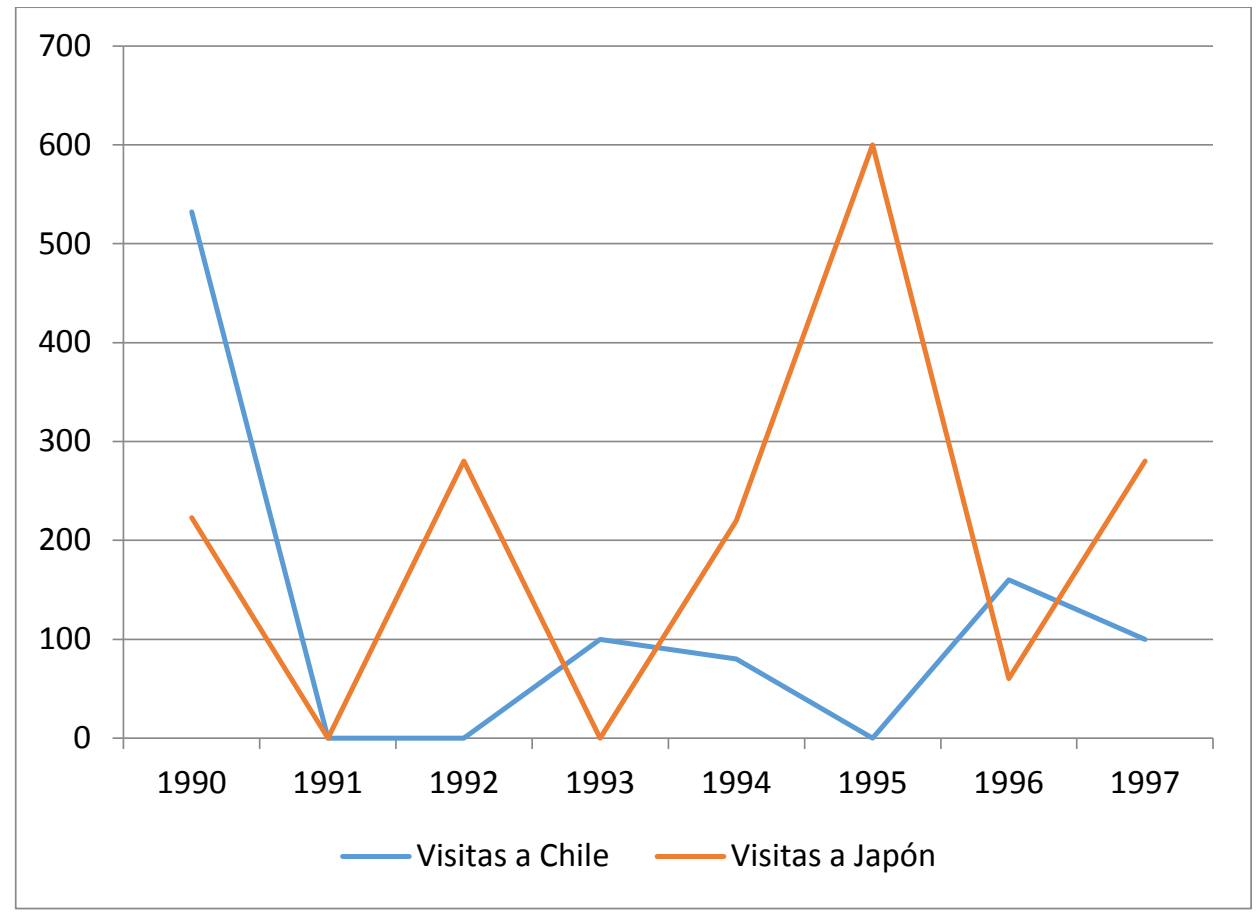

Fuente: elaboración propia de acuerdo con el registro de visitas bilaterales (Ross, 6 de junio de 2017).

\section{Avance de las relaciones bilaterales (1990-1993)}

Durante todo el período de negociaciones entre Japón y Chile, la clave no estuvo en lo que se dijo, sino que en lo que se hizo. Por ello, al revisar las memorias de ambos países, del Ministerio de Relaciones Exteriores para el caso chileno y el Diplomatic Bluebook del MOFA (sigla en inglés de Ministry of Foreign Affairs of Japan) para el caso japonés, es posible observar una primera etapa de las relaciones en el período 1990-1993, caracterizado principalmente por la apertura de la agenda política y económica.

La inauguración de esa década estuvo marcada por el comienzo de nuevos ciclos políticos para ambos países: Chile reinauguraba la democracia en marzo de 1990 y en noviembre del 
mismo año asumía el emperador Akihito en Japón. ${ }^{32}$ Así, la agenda bilateral se inició con la visita del senador Kaname Endo a la ceremonia de transmisión de mando del nuevo gobierno de Patricio Aylwin. Luego continuó con la visita a Japón de las nuevas autoridades chilenas: el ministro de Economía, Carlos Ominami; ${ }^{33}$ el director de Política Exterior, Carlos Portales; y el secretario general de Inversiones Extranjeras, Fernando Ibáñez. Luego, entre la segunda quincena de julio y la primera de agosto de 1990, tres delegaciones japonesas visitaron Chile, procedentes de la Japan International Development Organization (JAIDO), el MITI y la Agencia de Cooperación Internacional del Japón (JICA, por su sigla en inglés), lo que en el registro de visitas japonesas se evidencia con un peak en el Gráfico $\mathrm{N}^{\circ} 1$.

En lo cultural, el 24 de mayo de 1990, con motivo de los 30 años del terremoto de Valdivia (1960), ${ }^{34}$ el municipio de Minami-Sanriku (afectado por un tsumani que provocó este movimiento telúrico) descubrió un monumento conmemorativo con la figura de un cóndor mirando hacia la ciudad de Santiago, regalo del Gobierno chileno, ${ }^{35}$ y una placa conmemorativa que recordaba la amistad entre ambos países. ${ }^{36}$

En 1991, no hubo visitas y Japón fue el principal destino de las exportaciones chilenas, especialmente en productos no elaborados o semielaborados de la minería, sector forestal y pesca, cuya inversión fue apoyada por las $s \overline{o g o} s h \bar{o} s h a{ }^{37}$ De esta manera, Chile ingresó al Pacific Economic Cooperation Council (PECC), que se convirtió en una plataforma para el ingreso de Chile al APEC y más adelante al Foro de Cooperación América Latina Asia del Este (FOCALAE).

El 27 de julio de 1991, el Gobierno japonés apoyó económicamente la restauración de moáis en la Isla de Pascua, mientras que la empresa Tadano realizó una donación de fondos

32 La presencia de representantes en ambas ceremonias constituyó los primeros gestos políticos de ambos países en este período. Por parte de Japón, asistieron a la ceremonia de asunción de Patricio Aylwin realizada el 11 de marzo de 1990 -, el embajador especial y miembro de la Cámara Alta japonesa, Kaname Endo, el funcionario de MOFA, Yuji Takeya, y el embajador japonés en Chile, Rikiwo Shikawa. Por la parte chilena, asistió a la ceremonia de asunción al trono el entonces ministro de Relaciones Exteriores, Enrique Silva-Cimma.

33 Quien recibió el Cordón de la Orden del Sol Naciente en primer grado, una de las máximas distinciones del Imperio japonés, en reconocimiento a su trabajo por estrechar relaciones entre Chile y Japón.

34 El terremoto más fuerte registrado en el planeta desde que existen instrumentos de medición, cuya magnitud fue de 9,5.

35 En su pedestal lleva un texto para conmemorar la reconstrucción de ambos pueblos y países devastados por el terremoto y el tsunami de 1960, y un mensaje de amistad de la República de Chile al otrora pueblo de Shizugawa (actual Minami-Sanriku).

36 "Hace 30 años ocurrió un lamentable desastre que azotó a las costas del sur de Chile y también produjo un tsunami que arrasó con su pueblo, lo que genera un sentimiento de profunda empatía en los chilenos. Este monumento conmemorativo profundiza la amistad y el entendimiento mutuo entre el pueblo chileno y el japonés y es una prueba del compromiso de que nuestros lazos se seguirán fortaleciendo de aquí en adelante. Los chilenos sentimos un afecto especial por la larga historia de amistad y cariño entre ambos países. Embajada de Chile en Japón".

37 Las sōgō shōsha son compañías japonesas que comercian con una amplia gama de productos y materiales. Actúan como intermediarios, tanto en la logística, como en la exploración internacional de recursos. 
y equipo técnico para levantar 15 moáis, que hoy son la principal atracción turística de Ahu Tongariki. Ese mismo año, el Gobierno de Chile donó al municipio de Minami-Sanriku una réplica de un moái, que fue puesto al lado de la escultura del cóndor, y entre ellos se dispuso una placa conmemorativa con un mensaje del entonces presidente Patricio Aylwin. $^{38}$

El punto de inflexión en este período se produjo en noviembre de 1992, con la visita del presidente chileno a Japón, ${ }^{39}$ momento en que fue recibido por el emperador, el primer ministro Kiichi Miyazawa y los presidentes de las cámaras parlamentarias. Al igual que el año anterior, en 1993 la mayor cantidad de visitas registradas correspondió a las realizadas por chilenos, aunque se destaca en términos cualitativos la visita del príncipe y la princesa Hitachi a Chile en el mes de noviembre. Probablemente fue en este encuentro, realizado pocos días antes de la celebración de la APEC de 1993 en Seattle, cuando Japón formalizó su apoyo al ingreso de Chile a dicho foro. ${ }^{40}$

Respecto a las relaciones económicas bilaterales, el comercio entre ambos países se mantuvo bastante estable durante el período, evidenciándose una leve tendencia a la baja en las importaciones y exportaciones en 1993 (ver Tabla $\left.\mathrm{N}^{\circ} 1\right)^{41}$

Es importante destacar que en 1993 se produjo un quiebre del sistema político japonés, cuando el PLD perdió las elecciones que lo mantenía en el poder desde 1955 (aunque lo recuperó en enero de 1996). No obstante, esto no registró un impacto significativo en la agenda bilateral.

Revisado el período 1990-1993, se puede establecer que la característica principal de este fue la asimetría en los gestos diplomáticos entre ambos países, siendo Chile el actor más activo, mientras que, desde la perspectiva de la inserción económica internacional, el principal objetivo de la política exterior de Chile apuntó a conseguir su apoyo para ingresar a la APEC, meta que alcanzó en noviembre de 1993.

38 "Queridos habitantes de Minami-Sanriku: El moái es un símbolo de la cultura chilena y el hecho de que esté acá en Minami-Sanriku, es una prueba de los lazos de amistad de ambos pueblos, unidos por el mar, el mismo mar que aludía Pablo Neruda en su poema: 'La dentellada del mar muerde la abierta pulpa de la costa donde se estrella el agua verde contra la arena silenciosa"”.

39 Viajó con una delegación de parlamentarios y los ministros de Hacienda y Agricultura.

40 Cabe destacar que, entre los años 1990 y 1993, Chile otorgó la Orden Bernardo O’Higgins y la Orden al Mérito de Chile a 16 japoneses, entre los cuales se encontraban parlamentarios, diplomáticos y empresarios (Ross, 2001).

41 A diferencia de las relaciones comerciales entre Chile y China, las que se fueron incrementando incluso después de la crisis económica de 1997. Ver Tabla No 1 en artículo de Ross (2001). 
Tabla $N^{\circ}$ 1: Comercio de Chile con Japón (1990-1999) (países en porcentajes y total en millones de US\$)

\begin{tabular}{ccc}
\hline $\mathbf{A n ̃ o}$ & Importaciones & Exportaciones \\
\hline $\mathbf{1 9 9 0}$ & 568,6 & $1.388,2$ \\
\hline $\mathbf{1 9 9 1}$ & 646,3 & $1.644,0$ \\
\hline $\mathbf{1 9 9 2}$ & 965,1 & $1.707,4$ \\
\hline $\mathbf{1 9 9 3}$ & 882,6 & $1.502,3$ \\
\hline $\mathbf{1 9 9 4}$ & $1.007,1$ & $1.976,2$ \\
\hline $\mathbf{1 9 9 5}$ & 1.013 & $2.906,4$ \\
\hline $\mathbf{1 9 9 6}$ & 949,7 & $2.495,7$ \\
\hline $\mathbf{1 9 9 7}$ & $1.054,7$ & $2.675,8$ \\
\hline $\mathbf{1 9 9 8}$ & 994,6 & $1.959,3$ \\
\hline
\end{tabular}

Fuente: Boletín del Banco Central de Chile (cit. en Ross, 2001).

\section{Intensificación de la cooperación (1994-1995)}

Esta etapa coincidió con el inicio del gobierno de Eduardo Frei Ruiz-Tagle en marzo de 1994, a cuya ceremonia de asunción asistió una delegación japonesa. Durante este lapso, la agenda bilateral estuvo marcada por la candidatura y posterior elección de Chile como miembro no permanente del Consejo de Seguridad de Naciones Unidas para el período 1996-1997 y la paralela intensificación de la cooperación internacional de Japón hacia Chile. El país nipón oficializó su respaldo a la candidatura chilena en noviembre de $1994,{ }^{42}$ justo tras la visita de trabajo que el presidente Frei realizó a Japón y que luego repitió en 1995 con una gran delegación, ${ }^{43}$ lo cual se observa como el punto más alto del Gráfico $\mathrm{N}^{\circ} 1$.

42 Considerando que Japón solicitó que el Gobierno chileno respaldara sus posiciones en el Consejo respecto a la cuestión de la caza de ballenas, es posible plantear a modo de hipótesis que la cooperación japonesa estaba orientada a obtener el respaldo chileno en Naciones Unidas. Así, por ejemplo, la Memoria del Ministerio de RR.EE. chileno señala: "El tema ballenero es uno de los pocos puntos discrepantes en las relaciones bilaterales. La Cancillería nipona y la Agencia de Pesca Japonesa constantemente han tratado de cambiar la postura chilena a este respecto. Las autoridades japonesas han sido explícitas en señalar que la cooperación prestada para el desarrollo de los proyectos del Pueblo Artesanal de San Antonio y el Instituto de Investigación Pesquera, está vinculada a la búsqueda de soluciones intermedias que no perjudiquen los intereses japoneses en la Comisión Ballenera Internacional" (Ministerio de Relaciones Exteriores, 1994, p. 55).

43 Viajó junto al canciller Insulza, el ministro de Economía Álvaro García, seis parlamentarios, ocho rectores universitarios y más de cien empresarios. Fueron recibidos por el emperador, el primer ministro y la Liga 
Asimismo, en 1994 se realizó la primera reunión del grupo de rectores de ocho universidades chilenas con 14 rectores de universidades japonesas, celebrada en la Universidad de las Naciones Unidas en Tokio. En resumen, a contar de 1994 y hasta 1995, los contactos se intensificaron desde el lado chileno, correspondiendo al lapso en que se propuso la alianza estratégica del puente y en la que se trabajó intensamente en que esta diera sus frutos para el año del centenario. Sin embargo, detonó la crisis financiera asiática (1997), lo que afectó gravemente a la economía japonesa, evidenciándose un retroceso en las acciones que empujaban el TLC. ${ }^{44}$

\section{Repliegue de las relaciones bilaterales (1996-1997)}

En 1996 se realizó un encuentro entre académicos de las universidades japonesas y chilenas en Osaka, donde se discutió la posibilidad de un intercambio universitario más amplio, lo que no llegó a prosperar. Ese mismo año, el primer ministro Ryūtarō Hashimoto visitó Chile $^{45}$ con el objetivo de difundir y consolidar la necesidad de cooperación bilateral para la construcción de una alianza entre Japón y Chile en la nueva era:

El compromiso adquirido por ambos gobiernos, en las personas de sus jefes de Estado, construyó uno de los puntos más altos de las relaciones bilaterales, en el que ambos países le dieron a su contraparte el lugar de socio estratégico, excediendo con creces lo que ambos Gobiernos habían establecido en sus propias declaratorias de política exterior. Por lo tanto, la cita de 1994 entre ambos jefes de Estado se transformó en un hito histórico que dio comienzo a la hipótesis del puente: visión, imagen y símbolo de lo que se pretendía construir entre ambos países (Ross, 2012).

En 1997, y con ocasión del centenario de las relaciones bilaterales, se realizaron distintas actividades a nivel oficial y privado. A fines del mes de agosto, el presidente Frei viajó a Japón en el buque Escuela Esmeralda, el que transportó una gran bandera japonesa que el Gobierno de Chile donó para conmemorar esta importante celebración. Hacia la tercera semana de septiembre, el gesto fue retribuido por medio de la visita de los príncipes Hitachi a Chile.

Entre los días 30 de septiembre y 2 de octubre de 1997 se celebró por primera vez en Chile, y en un país latinoamericano, la Reunión General del Consejo de Cooperación Económica del Pacífico, PECC XII, como culminación de un período que se inició en 1995 y que se extendió hasta octubre de 1997, durante el cual la presidencia de dicho foro correspondió a

de Parlamentarios Amigos de Chile. Además, el Gobierno chileno reiteró su apoyo para que Japón se convirtiese en miembro permanente del Consejo de Seguridad.

$44 \mathrm{Si}$ bien no se consideró en el Gráfico $\mathrm{N}^{\circ} 1$, tanto en la agenda de fuentes japonesas como en la agenda de fuentes chilenas, 1998 es uno de los años de menor intensidad en las relaciones bilaterales.

45 Esta fue la segunda visita de un primer ministro japonés; la primera corresponde a la de Nobusuke Kishi en 1959, quien es abuelo del actual primer ministro, Shinzo Abe. 
Chile. ${ }^{46}$ Producto de esta reunión, se produjo un aumento de las visitas japonesas a Chile, aunque estas no respondían necesariamente a un avance de la alianza estratégica. En este período, las exportaciones se mantuvieron estables, mientras que las importaciones variaron a la baja, pese a que en 1997 se recuperaron (Tabla $\mathrm{N}^{\circ} 1$ ).

Finalmente, la crisis económica de 1997 significó para Japón la aceleración de un proceso complejo que se había iniciado con la crisis de burbuja y que coincidió con el ascenso de China a la cima de la economía mundial, en detrimento directo de Japón. Frente a esto, el empresariado chileno, seducido por el tamaño de su economía, comenzó a mirar al dragón asiático como un nuevo socio comercial. Desplazaba de esta manera su foco de interés, centrándolo ahora en China en desmedro de Japón, lo que generó una tensión entre los principios (amistad) y los intereses (económicos) de la política exterior de ambos países. Lo anterior provocó que este proyecto no se concretara en el tiempo ni de la forma en que se había planeado inicialmente, lo que para Chile significó perder la posibilidad de tener una alianza estratégica con una de las mayores economías mundiales durante la década de 1990, oportunidad que nunca tuvo antes y que no volvió a tener. ${ }^{47}$

La jerarquización de las visitas durante el período 1990-1997, junto con la revisión histórica de este, permitió comprender las claves de la relación bilateral, lo que resultó esencial para la construcción de un relato que describe y analiza las claves de una relación de dos países con desarrollos tan diferentes como Japón y Chile. El avance, intensificación y repliegue en las relaciones bilaterales en el marco de un escenario adverso posterior a la Guerra Fría, así como los gestos (visitas) y símbolos (moáis, bandera) implicados en estos acercamientos, evidenciaron el interés de ambos países por concretar la idea del puente. No obstante, la revisión de la agenda muestra también la carencia de la visita de profesionales técnicos (burocracia de ambos países), cuestión que pudiera haber constituido la fuerza de trabajo que concretara la alianza estratégica. Si bien el papel del empresariado de ambos países fue muy importante, especialmente el rol jugado por el Capítulo Chileno del Comité Empresarial Chile-Japón, esto no fue suficiente para concretar el acuerdo.

\section{Conclusiones}

La revisión de las relaciones comerciales entre Japón y Chile permite advertir una experiencia histórica que se puede denominar de asimetría virtuosa, la cual ha proporcionado beneficios para ambas partes y que en la década de 1990 derivó en el entusiasmo por impulsar una alianza estratégica compleja (económica, política y

46 Esta reunión no se consideró en la construcción del gráfico de visitas bilaterales, pues el número de visitas generaba una sobrerrepresentación en los indicadores, y con ello afectaba el patrón de visitas.

47 Si bien en 2007 ambos países firmaron un TLC, las condiciones históricas y económicas de los noventas nunca se repitieron. 
geográfica) entre ambos países. En la base de la propuesta estaba el interés de Japón por dinamizar su economía y potenciar el intercambio político y académico, así como el de Chile por avanzar en su reinserción internacional luego de 17 años de dictadura militar. Esto, movilizó a los Gobiernos, empresariados y académicos de ambos países en pos de establecer un vínculo estratégico que debía convertirse en un puente entre los países de Asia Pacífico y los del MERCOSUR.

No obstante, la posición de liderazgo regional y mundial de Japón fue impactado por la crisis deflacionaria de la década de 1980 y por la crisis asiática de la siguiente década. Ambos hechos constituyeron un punto de inflexión en la historia mundial reciente, pues significó el estancamiento japonés, el fin de la amenaza nipona a la hegemonía estadounidense, el ascenso de China y, con ello, el cambio en el eje de potencias.

Hechos como estos tensionaron las variables externas e internas japonesas que, con la influencia del aumento de flujos comerciales en la Cuenca del Pacífico, así como con la crisis económica de los noventas, inevitablemente influyeron en la toma de decisiones del Gobierno japonés y cambiaron su política exterior.

En este contexto, Japón buscó socios fuera de la región para contrarrestar el balance de poder, así como para desarrollar alianzas estratégicas que le permitieran acceder a materias primas. Con esa perspectiva vio a Chile como un posible socio comercial que le permitiría ser la puerta de entrada a las economías de los países del MERCOSUR. Si bien ambos países tenían un siglo de relaciones bilaterales bastante pragmáticas (especialmente durante los setentas y ochenta del siglo pasado), también habían construido relaciones de confianza y lealtad, basadas en la estabilidad política chilena y en el respeto a las reglas del juego. En este escenario, el rol del empresariado japonés y chileno fue esencial para el avance de la propuesta.

Por otra parte, la revisión del patrón de visitas durante el período de estudio muestra que los distintos gestos políticos y simbólicos evidencian el interés común por dinamizar ambas economías y aumentar el intercambio de bienes, por lo que resulta complejo explicar por qué tanto esfuerzo por alcanzar un objetivo tan relevante fue insuficiente.

Una primera explicación posible es que este proyecto se frustró por la escasa inmigración japonesa en Chile. Esto generó una debilidad en las relaciones bilaterales, especialmente en lo referido a la construcción de lazos más profundos que empujaran a ambas naciones hacia una alianza casi natural que debía contar con el apoyo de descendientes japoneses en Chile (tal como sucedió en Brasil, México y Perú).

Una segunda explicación es que la revisión de las visitas bilaterales muestra una falta de profesionales técnicos (planta burocrática) que permitiera el desarrollo de informes y propuestas para la concreción de los planes. 
La tercera explicación podría estar basada en la idea de que Chile creció y se desarrolló en los noventas, y el objetivo de su política exterior apuntó a la reinserción económica. De ahí que las decisiones que tomó se guiaron de manera pragmática $\mathrm{y}$, ante la apertura del mercado chino (y el descenso económico japonés), optó por impulsar este mercado. Por extensión, es posible que los tomadores de decisiones de entonces no hubieran tenido plena conciencia de la envergadura de lo que estaba en juego (Ross, 2012).

Estas explicaciones evidencian una tensión entre los principios (lealtad) e intereses (económicos) de ambas naciones, dado que las relaciones de amistad sostenidas hacían pensar que la alianza se concretaría en la década de 1990. Sin embargo, la crisis económica japonesa hizo que la economía chilena se interesara en el mercado chino, haciendo fracasar de esta manera la idea del puente y, con ello, la oportunidad de Chile de asociarse estratégicamente con la segunda potencia del mundo, una alianza que se realizaría sin una subordinación política ni bajo algún sometimiento ideológico.

Si bien el objetivo de firmar un TLC se alcanzó en 2007, las condiciones económicas de la década anterior no se repitieron. Japón había entrado en una crisis económica a fines de los ochentas, que se profundizaría en los noventas. Las consecuencias de este declive económico le hicieron perder su liderazgo regional y mundial, dando paso a la emergencia de un nuevo líder económico: China, que se convirtió en una atracción económica muy poderosa para los países latinoamericanos ante la cual Chile no fue la excepción. De esta forma, en el mismo período en que Chile trabajaba fuertemente en la reinserción política y económica, Japón ingresó a una fase de inestabilidad que dilató las conversaciones y cuya consecuencia inevitable fue el repliegue del plan estratégico bilateral y la idea del puente entre ambos países.

\section{Referencias bibliográficas}

Amparo, D. (2005). Cooperación internacional en la Cuenca del Pacífico. APEC: la génesis de una institución. México y la Cuenca del Pacífico, 8(24), 123-129.

Belém, D., Damascena, L. y Mendonça, C. (2015). La política exterior del Chile democrático: el papel de los empresarios y el surgimiento de la "diplomacia paralela". Cuadernos Americanos, 154(4), 101-123.

Braudel, F. (2005). La historia y las ciencias sociales. Madrid: Alianza Editorial.

Burges, S. (2014). Latin America and the Shifting Sands of Globalization. Londres: Routledge.

Casson, M. (2010). Entrepreneurship. Theory, Networks, History. Northampton, Mass.: Edward Elgar Publishing. 
Comité Empresarial Chile-Japón. (1994). Actas de la 15ª Reunión del Comité Empresarial Chile-Japón (Anexo A), 17 y 18-noviembre, Tokio, Japón.

Cooney, K. (2015). Japan's Foreign Policy Since 1945. Nueva York: Routledge.

Dickson, P. H. y Weaver, K. M. (1997). Environmental determinants and individual-level moderators of alliance use. Academy of Management Journal, 40(2), 404-425.

Faust, J. (2004). Latin America, Chile and East Asia: Policy- Networks and Successful Diversification. Journal of Latin American Studies, 36(4), 743-770.

Grant, R. y Baden-Fuller, C. (2004). A knowledge accessing theory of strategic alliances. Journal of Management Studies, 41(1), 61-84.

Gutiérrez, H. (2005). Chile and Pacific Asia: the economic connection. En J. Faust y M. Mols, Latin America and East Asia. Attempts at diversification. Seúl: Münster.

Hollis, M. y Smith, S. (1990). Explaning and Understanding International Relations. Oxford: Oxford University Press.

Huguet, M. (2001). Historia del tiempo presente e historia de las relaciones internacionales. Revista Ayer, 42, 43-69.

Jarufe, J. P. (BCN). (2018). La República Popular China y sus vínculos con Chile. Departamento de Estudios, Extensión y Publicaciones de la Biblioteca del Congreso Nacional de Chile, 7 de mayo. [En línea]. Recuperado de https://www.bcn.cl/obtienearchivo?id=repositorio/10221/25270/1/La_Republica_Po pular_China_y_sus_vinculos_con_Chile_Mayo_2018.pdf

Kagami, M. (2011). Tendencias recientes de la integración asiática y la participación de Japón. México y la Cuenca del Pacífico, 14(42), 15-36.

Kamikubo, M. (2008). Bureaucratic behaviour and policy change: a case of Japan's financial market reform in the 1990s as an implication for the study of asian finacial integration. Global COE Program, Global Institute for Asia Regional Integration (GIARI), Waseda University, 7 de enero.

Keohane, R. y Nye, R. (1977). Power and Interdependence. Londres: TBS The Book Service.

Laborde, A. (2006). La política migratoria japonesa y su impacto en América Latina. Migraciones internacionales, 3(3), p.155-161

Marris, E. y Overlana, M. (1997). The History of the Pacific Basin Economic Council 1967 to 1997, Bridging the Pacific. Wellington: Pacific Basin Economic Council.

Masterson, D. y Funada-Classen, S. (2004). The Japanese in Latin America. Illinois: University of Illinois Press.

Minería Chilena (2014). El 54\% de la inversión japonesa en Chile es en minería. Minería Chilena, 13 de febrero. Recuperado de http://www.mch.cl/2014/02/13/el-54-de-lainversion-japonesa-en-chile-es-en-mineria/\# 
Nagata, Y. (2017). Bureaucracy in the Power-Dependence of Domestic Politics and Linkage in Foreign Police. [Revisión de video, producido por International Academic Forum (IAFOR). The IAFOR International Conference on the Social Sciences, Dubai Conference Series 2017 Virtuals. Recuperado de https://papers.iafor.org/submission35119/]

Nagatomi, Y. (1988). Masayoshi Ohira's Proposal: To Eolve the Global Society. Tokio: Foundation for Advanced Information and Research.

Nash, N. (15 de abril de 1993). Japan's Backdoor to the West, New York Times, p. 1.

Pezzola, A. (2016). Japanese access to Mexico: the structure of the Japan-México FTA. México y la Cuenca del Pacífico, 5(15), 23-50.

Rodríguez, I. y Leiva, D. (2013). El soft power en la política exterior de China: consecuencias para América Latina. Polis, 12(35), 497-517.

Ross, C. (2001). El Comité Empresarial Chile- Japón: de la liturgia al libre comercio, 19791999. Diplomacia, 86, 89-111.

Ross, C. (2002). Relaciones entre Chile y China: treinta años de relaciones atípicas, 19792000. Si Somos Americanos. Revista de Estudios Transfronterizos, 3(2), 33-48.

Ross, C. (2007). Chile y Japón: 1973-1989. De la incertidumbre a la alianza estratégica. Santiago: LOM.

Ross, C. (2012). La política de Chile hacia Japón 1990-2010: la relevancia del largo plazo. En M. Aratza y C. Ross, La politica exterior de Chile: del aislamiento a la integración global (pp. 773-794). Santiago: Ril Editores-USACH.

Ross, C. (2013). Auge y caída de Japón en Chile, 1897-1943. Estudios Políticos, 43, 156179.

Ross, C. (2014). Chile y Japón durante los mil días de Allende, 1970-1973: El camino lateral. Universum, 29(2), 277-290.

Ross, C. (6 de junio de 2017). Entrevista para proyecto Fondecyt № 1140244.

Ross, C. (2018). Chile-Japón, 1990-2007: avances y repliegues en una alianza estratégica fallida. Diálogos Andinos, 53, 101-117.

Ross, C. y Leiva, S. (2017). La política de Chile hacia Bolivia, 1990-2009: ni coordinación política, ni cooperación económica. Estudios Políticos, 50, 17-41.

Stallings, B. (2009). Chile: a pioneer in trade policy. En M. Solís, B. Stallings y S. Katada, Competitive Regionalism FTA Diffusion in the Pacific Rim (pp. 118-138). Londres: Palgrave Macmillan.

Stallings, B. y Székely, G. (1994). Japón, los Estados Unidos y la América Latina: ¿hacia una relación trilateral en el hemisferio occidental? México D.F.: Fondo de Cultura Económica. 
Tanaka, N. (2002). Economic restructuring and trade policy in Japan: Is the new WTO round a threat or opportunity? From the Doha to Kananakis Conference at Toronto, March 1-2 (pp. 1-5). Toronto: York University. Recuperado de http://www.yorku.ca/drache/talks/pdf/doha_tanaka.pdf

Toro, L. (2018). La política exterior de seguridad japonesa. Historia y teoría. Santiago: Ariadna.

Valenzuela, M. y Varas, A. (1993). El creciente papel económico de Japón en Chile. Cono Sur, 12(1), 20-25.

Wilhelmy, M. y Lazo, R. (1997). La estrategia bilateral de Chile en el Sudeste asiático. Revista de Ciencia Política, 19(1), 37-60.

Williams, A. (2012). History and international relations. Contrasts and comparaisons. En A. Williams, A. Hadfield y S. Rofe, International History and International Relations (pp. 7-32). Nueva York: Routledge.

Yoshimatsu, H. (2006). The politics of Japan's free trade agreement. Journal of Contemporary Asia, 36(4), 479-499.

\section{Archivos}

Memorias del Ministerio de Relaciones Exteriores de Chile (1990-1998). 